\title{
Determinants of Responses to Oral Vaccines in Developing Countries
}

\author{
David A. Sack ${ }^{\text {a }}$ Firdausi Qadri $^{\text {b }}$ Ann-Mari Svennerholm ${ }^{c}$ \\ ${ }^{a}$ Department of International Health, Johns Hopkins Bloomberg School of Public Health, Baltimore, Md., USA; \\ ${ }^{\mathrm{b}}$ Laboratory Science Division, International Centre for Diarrhoeal Disease Research Bangladesh, Dhaka, Bangladesh; \\ 'Department of Microbiology and Immunology, University of Gothenburg, Gothenburg, Sweden
}

\begin{abstract}
Key Words
Oral vaccines $\cdot$ Cholera $\cdot$ Rotavirus $\cdot$ Typhoid $\cdot$ Polio $\cdot$

Shigellosis $\cdot$ Immune response $\cdot$ Malnutrition $\cdot$ Zinc $\cdot$

Vitamin A $\cdot$ Breastfeeding
\end{abstract}

\begin{abstract}
Oral vaccines which are intended for global use do not necessarily induce the same immune responses in all children worldwide. In fact, several vaccines often induce less frequent and lower mean antibody responses in children in developing countries, suggesting that the vaccines may be less protective among children in these areas. Though the reasons for this less vigorous response are not completely understood, it appears that nutrition-related factors, including both protein-calorie and micronutrient malnutrition, are important aspects in understanding the hyporesponsiveness seen in these children. Related issues including breastfeeding, interference from maternal placental antibodies, intestinal parasitic infections, intestinal mucosal damage and possibly maternal malnutrition during pregnancy also appear to be important. Vaccines designed for oral administration will need to be adjusted to these potential problems in order to maximize benefits for all children. Oral vaccines, when given to children in developing countries, may require higher doses of vaccine, booster doses, calorie, micronutrient and vitamin supplements, withdrawal of breast milk before vaccine administration, deworming medications or other measures to realize their full benefit.
\end{abstract}

Copyright $\odot 2008$ Nestec Ltd., Vevey/S. Karger AG, Basel
(C) 2008 Nestec Ltd., Vevey/S. Karger AG, Basel 0517-8606/08/0662-0071\$24.50/0

Fax +4161306 1234 E-Mail karger@karger.ch www.karger.com
Accessible online at: www.karger.com/ane

\section{Introduction}

Vaccination is one of the most powerful preventive strategies for improving the health of people throughout the world. Children in most developing countries are especially vulnerable to infectious diseases as evidenced by their high infant and child mortality rates, with pneumonia and diarrheal diseases being the largest causes of these potentially fatal infections. Vaccines are becoming available for many of these infections. In addition to the standard package of vaccines for diphtheria, pertussis, tetanus, polio, measles and tuberculosis, newer vaccines for hepatitis B, Haemophilus influenzae type B, are now commonly included in the Expanded Programme for Immunizations. Even newer vaccines for Streptococcus pneumoniae and rotavirus are entering routine use in some developing countries and others for typhoid and cholera will likely be used in some countries. Of these, several vaccines are given by the oral route including oral polio vaccine (OPV), rotavirus, cholera and some typhoid vaccines. In addition, new vaccines are being developed for enterotoxigenic Escherichia coli (ETEC) and Shigella spp., which will likely be given orally as well.

Experience with several oral vaccines has shown that immune responses may not be as robust or consistent when given to children in developing countries as when given to children in industrialized countries. Among this list of oral vaccines, the ones for bacterial infections (cholera, ETEC, typhoid, shigellosis) will likely only be used 
for children in developing countries or for travelers since these are the persons at risk. Although polio vaccine is routinely given throughout the world, nearly all industrialized countries now use injectable vaccine rather than OPV. The new vaccines for rotavirus are given orally, and since this is an infection which occurs in all geographic regions, the same formulation will be used globally.

This paper reviews studies which have evaluated oral vaccines in developing countries, especially in children, and nutritional and other factors that may explain the lower immunogenicity and protective efficacies often recorded in children in the developing world compared with industrialized countries. Also included are studies which have examined different variables which might improve a given vaccine for use in developing countries.

This paper concentrates on oral rather than injectable vaccines since most evaluations of injectable vaccines find that children in developing and industrialized countries respond in a similar manner. Only when children have extremely severe malnutrition are the immune responses to injectable vaccines blunted [1]; however, oral vaccines present some special issues with regard to nutritional status.

To conduct this review, we searched PubMed for the following terms: OPV, rotavirus vaccine, typhoid vaccine, cholera vaccine and Shigella vaccine, and we restricted our search to those papers that were related to immunogenicity or efficacy studies in developing countries, especially related to nutritional factors. Since several of the vaccines have specific names, we included them in our search, e.g., CVD103-HgR and Peru-15 cholera vaccine, Ty21a typhoid vaccine, SC602 Shigella vaccine, RIX4414, RotaRix ${ }^{\circledR}$, RotaTeq ${ }^{\circledR}$ and RotaShield ${ }^{\circledR}$ rotavirus vaccines. In some cases, unpublished data and results of such studies have been included where appropriate.

\section{Oral Polio Vaccine}

The formulation of OPV, being a trivalent vaccine, is adjusted such that each of the vaccine serotype strains has a high probability of inducing protective immune responses against each of the 3 serotypes following 3 doses of vaccine. Immune responses are measured as neutralizing antibody titers using a tissue culture assay, and a titer of $\geq 1: 8$ is said to be protective. Studies from industrialized countries have found that immunization with 3 doses of the trivalent OPV induces protective antibody titers in $>95 \%$ of children [2]. However, when the vaccine was given to children in developing countries, it stimulated lower and less reliable antibody responses [3-7]. This was especially true for serotype 3 .

The reasons for the less consistent response among children in developing countries are not clear; however, most studies have concentrated on the potential role of competing enteric virus infections which may interfere with the vaccine virus infection of the intestine and the possibility that the vaccine virus is being neutralized by breast milk antibodies. Some studies have also evaluated the role of nutritional status, e.g., the effect of vitamin A supplementation on the immunogenicity of OPV.

Data are conflicting with regard to interference from other enteric viruses, with some studies finding an association $[7,8]$ while another was unable to confirm this association, but did find poor responses even in children with no other enterovirus infection [5]. The study by Triki et al. [7] also found an association of poorer responses with lower socioeconomic status, and within the summer season, the latter factor was likely a surrogate for other enterovirus infections.

There is strong evidence that breast milk antibodies can interfere with OPV by neutralizing the vaccine virus and preventing infection of intestinal mucosa [9]. This observation was supported by studies from Uganda [10] and by experimental studies in which milk formula containing neutralizing antibodies at various times before or after vaccination was found to interfere with the vaccine take [11]. When the antibody-containing formula was given within $2 \mathrm{~h}$ of vaccination, it inhibited vaccination, but not if the formula was separated by $6 \mathrm{~h}$. Separating breastfeeding from vaccination by $6 \mathrm{~h}$ may not be practical in many developing countries, and one study in India did not find an association between breastfeeding and vaccine immunogenicity [12].

A study from Bangladesh found a reduced immune response to OPV if the vaccine was given at a time when the child had diarrhea [13]. This suggested that the rapid transit time associated with diarrhea led to less colonization by the live vaccine virus; however, other confounding factors, such as poor nutrition in children with diarrhea, could not be ruled out.

Strategies to improve immune responses to OPV have generally focused on increasing the number of vaccine doses $[14,15]$, although this is not always successful [6]. In a study in which more doses failed to improve the immunogenicity of OPV, an injectable polio vaccine was successful [6]. One study from India found that vitamin A supplements to the mothers shortly after delivery, and to the babies at the Expanded Programme for Immuniza- 
tions visits, increased the titers of serotype 1 following OPV, but did not affect responses to other serotypes [16]. From a practical viewpoint, national programs have attempted to give multiple additional doses of OPV, often combined with vitamin A administration, at National Immunization Days, which have been successful in eradicating polio from nearly all countries.

Reduced immunogenicity of OPV in developing countries is well recognized, and the experience with OPV is relevant to other oral vaccines. It seems that other oral vaccines may also be less immunogenic when given to children in developing countries, and that the lessons learned from OPV may also apply. As the number of oral vaccines expands, other factors are being examined, especially those related to nutrition.

\section{Rotavirus Vaccines}

Several live oral rotavirus vaccines have been evaluated in both developing and developed countries including the bovine RIT vaccine, a monovalent bovine WC3, the tetravalent rhesus rotavirus vaccine (RRV-TV or RotaShield), a human attenuated vaccine, RIX4414 (RotaRix), and a pentavalent WC3 reassortant vaccine (RotaTeq). RIT and the monovalent WC3 were early vaccines which appeared to be promising but then failed to be sufficiently protective when used in developing countries [17-20]. RRV-TV was efficacious in Europe, the US and Venezuela, but appeared to be less protective in other developing countries [21-25]. A reanalysis of the results of the trials in Peru and Brazil suggested that the efficacy of the vaccine was actually higher than originally appreciated when restricted to those which had more severe diarrhea [26]. Reasons for the lower efficacy in developing countries were not explained but it seemed that these rotavirus vaccines generally yielded a lower efficacy when tested in developing countries.

Following the withdrawal of RotaShield from the US market, the newer rotavirus vaccines, RotaRix and RotaTeq, were developed and found to be safe and effective in developed and/or middle income countries and in the US, Europe, Latin America and Asia [27, 28]. However, they had not been tested in very poor countries, and thus, studies were initiated to determine their safety, immunogenicity and efficacy in very poor countries of Asia and Africa which are eligible for funding by GAVI (Global Alliance for Vaccines and Immunization). RotaRix has now been found safe and immunogenic in South African and Bangladeshi children, but its immunogenicity may

Vaccine Responses in Children in

Developing Countries be somewhat diminished compared with children in other countries [D. Steele and K. Zaman, pers. commun.].

Reasons for the somewhat lower seroconversion rates for rotavirus vaccines in developing countries are not established. Speculation has included interference from breast milk which might have neutralized the vaccine virus, or high levels of maternal transplacental antibodies which, likewise, might have blunted the response. Thus, in an early meta-analysis, it was shown that breastfed children receiving an RRV (serotype 3) seroconverted in significantly lower frequency than bottle-fed children (48 vs. $70 \%$; $p<0.005$ ) [29]. However, subsequent studies from the US have not supported these findings [30]. Speculation has also included a nonspecific role for malnutrition or a specific micronutrient deficiency. Thus, when evaluating the protective efficacy of the RRV-TV vaccine according to the nutritional status in infants participating in a phase III trial in Peru, a greater efficacy was found among children reaching a weight for age $Z$ score of more than -1 and a height for age $Z$ score of more than -1 compared with children with lower scores [31]. However, in a recent report from different countries in Latin America, vaccine efficacy induced by the human rotavirus vaccine RIX4414 was similar in well-nourished and malnourished infants [32]. One study from Bangladesh demonstrated that supplemental zinc did increase the geometric mean titers of infants immunized with RotaShield [A. Baqui, pers. commun.].

\section{Typhoid Vaccine}

There are 2 licensed typhoid vaccines which are commonly used, an oral live attenuated bacterial vaccine, Ty21a (Vivotif ${ }^{\circledR}$ ), and an injectable polysaccharide vaccine $\mathrm{Vi}$ (Typhim $\mathrm{Vi}^{\circledR}$ ). $\mathrm{Vi}$ is given to children older than 2 years and to adults, but is not given to young children and infants, since this type of polysaccharide antigen is not immunogenic in the youngest age groups. A conjugated Vi typhoid vaccine was more immunogenic and protective when tested in Vietnam [33]. Unfortunately, this conjugate vaccine is not yet available.

Ty21a is approved for children $\geq 5$ years old, but has not been extensively tested in younger children. One study involving a small group of children $(n=18)<24$ months of age found the vaccine to be safe; however, there was no measurable immune response in these children [34]. A separate, larger study of 634 children 2-6 years old in Thailand found it to be safe and immunogenic with similar take rates in all ages, without a significant drop 
in take rates in the youngest age groups [35]. Studies will be initiated soon to determine the immunogenicity of a liquid formulation of this vaccine in Bangladeshi children, 2-5 years of age, to relate immune responses to the nutritional status of the children and the possible benefit of zinc supplementation. Unfortunately, this oral vaccine has not been tested for efficacy in the young age groups ( $<5$ years old), especially in light of the high attack rates in young children in some South Asian countries.

\section{Live Oral Cholera Vaccine}

There are currently 2 attenuated oral cholera vaccines, CVD103-HgR (Orochol ${ }^{\circledR}$ ) and Peru-15 (CholeraGarde ${ }^{\circledR}$ ), which have been extensively tested in subjects of both developing and developed countries. CVD103-HgR is licensed, but Peru-15 is still under development [36].

CVD103-HgR was normally given at a dose of $10^{8}$ organisms per dose along with a buffer solution containing sodium bicarbonate and ascorbic acid designed to protect the live bacteria from stomach acid. This formulation was safe, immunogenic and protective in North American volunteers. When the vaccine was given to residents in Chile and Indonesia, it was less immunogenic than expected, so the dose was increased by 1 log to improve its immunogenicity [37-40]. Unfortunately, even the higher dose of vaccine did not demonstrate protection in the only controlled field trial of the vaccine in Indonesia [40]. The contrast between the high efficacy when tested in volunteers in North America and the low efficacy in Indonesia is poorly understood, but may be related to preexisting natural immunity to cholera, and hence, a decreased 'take' by the live vaccine. Furthermore, the rates of cholera were unusually low during the early phases of the trial, and the short-term efficacy could therefore not be determined.

Additional factors which may have reduced the immunogenicity of CVD103-HgR in Indonesia include small bowel overgrowth, intestinal parasitic infections and undernutrition. Thus, one study has demonstrated that small bowel bacterial overgrowth, as detected by a breath hydrogen test, was associated with a somewhat diminished response to CVD103-HgR in Chile [41]. This finding suggests that abnormal intestinal mucosa (e.g., tropical enteropathy) commonly associated with small bowel overgrowth, may be partly responsible for the less robust response in these subjects. Another study examined the potential role of infection with Ascaris lumbricoides and found that treating this parasitic infection prior to vac- cination improved the cell-mediated immune response to the vaccine [36]. The effect of treatment with albendazole and secnidazole on the take rates of live oral typhoid or killed oral cholera vaccines are being planned for toddlers with parasitic infections in Bangladesh.

The other live oral cholera vaccine Peru-15 was found to be safe, immunogenic and protective in US volunteers [42-44] at doses of $2 \times 10^{8}$. This dose has now been tested in Bangladeshi adults and children. Although the take rate at this dose has been high in Bangladesh in all age groups, the geometric mean titers in the youngest age group (9-23 months) were lower than in older subjects, and a dose 1 log less was distinctly less immunogenic [45]. The vaccine has not been evaluated in US children for comparison, so it is not possible to determine if the lesser response in the youngest Bangladeshi children is related to age or to other factors.

Of interest, Peru-15 is normally given with a buffer consisting of sodium bicarbonate and ascorbic acid, similar to the buffer used with CVD103-HgR. In one study, when given to US volunteers with a buffer of rice syrup, bicarbonate and citrate, the geometric mean antibody titers were higher than when the standard buffer was used, suggesting that factors other than the dose of the vaccine may be important [46]. In this study comparing different buffers, the control group receiving the vaccine with no buffer (saline only) had a very poor antibody response indicating that acid neutralization is critical to the immunogenicity of this live, acid-sensitive, oral vaccine.

Studies are planned to evaluate Peru- 15 when given concurrently with measles vaccine to rule out possible interference between the cholera vaccine and the measles vaccine in infants $<1$ year of age. This type of study is needed since it seems likely that the 2 vaccines may need to be administered at about the same age.

\section{Killed Oral Cholera Vaccines}

There are 2 killed oral cholera vaccines. The killed whole cell with recombinant $B$ subunit of the cholera toxin $\left(\mathrm{WC} / \mathrm{rCTB}\right.$; Dukoral $\left.{ }^{\circledR}\right)$ is licensed in more than 50 countries $[47,48]$, and another similar whole-cell vaccine without B subunit is licensed in Vietnam [49] and is currently being tested in India. The whole-cell component of the vaccine is acid stable; thus, it does not require a buffer to protect it from gastric acid. However, the B subunit is acid labile and does require a buffer. Dukoral is safe, immunogenic and protective as documented from studies in Bangladesh, Peru and Mozambique [50-54]. The Ban- 
gladesh study illustrates its protective benefit in an area with high rates of malnutrition, and the study from Mozambique confirms its benefit in an area with high rates of HIV infection.

Though the vaccine was protective in these different geographic areas, the antibacterial (vibriocidal) responses were less frequent in young children (2-5 years) compared with older children and adults [55], and more doses were required to achieve a higher take rate [56]. Similarly, the magnitude of the antitoxin responses was lower in the young age groups ( $2-5$ years) than in older groups [57]. Comparison of immune responses with the cholera toxin B whole-cell vaccine (CTB WCV) in age-matched children (down to 1 year of age) in Sweden and Nicaragua using calibrated serological techniques found that although the Swedish children had lower preimmunization titers, they developed more pronounced antitoxin as well as vibriocidal antibody responses to the vaccine [58]. However, it is not clear if these differences were related to nutrition or other factors such as previous exposure to these cholera antigens.

The association between undernutrition and the colonization with Vibrio cholerae and serologic response to orally given CTB was studied in Bangladeshi children in the 1980s [59]. These studies revealed that undernutrition (determined as $<70 \%$ of the reference weight for age) was not associated with colonization, disease or the duration or severity of cholera. Moreover, undernourished children did not differ from well-nourished children in their serologic response to colonization, to disease or to CTB given orally. It was concluded that the good response to the CTB component among undernourished children was of particular importance when considering the use of oral cholera vaccines in areas where undernutrition is common. However, in recent reanalyses of previous studies in Bangladesh when severely malnourished children were given Dukoral, $\geq 4$-fold increased vibriocidal immune responses were significantly less frequent in malnourished children (less than -2 SD height for age Z score) than in better nourished children [Ahmed et al., unpubl.]. Thus, it may be that a very potent immunogen such as CTB may be less affected by the nutritional status of the child than the bacterial antigens, predominately lipopolysaccharides, which induce vibriocidal immune responses.

Some studies have attempted to improve the immunogenicity of the killed WC/rCTB vaccine with the use of micronutrients, with the hypothesis that some children may not be responding optimally due to micronutrient deficiency. Results of these studies are mixed. In one study, children were supplemented with either vitamin A or zinc, or a combination of both vitamin $\mathrm{A}$ and zinc. Children who were supplemented with zinc had higher vibriocidal seroconversion rates than the other children, but strangely, had lower rates of antitoxin responses [60, 61]. By contrast, vitamin A did not change either of these serological responses. In more recent studies, there was a highly significant effect on the vibriocidal immune responses to Dukoral in Bangladeshi children 9-18 months of age who were supplemented with zinc for 42 days (20 $\mathrm{mg} /$ day), starting 3 weeks before onset of vaccination [Ahmed et al., submitted]. Seventy-seven percent of the supplemented children mounted a $\geq 4$-fold increased vibriocidal serum antibody response as compared with only $56 \%$ of the nonsupplemented children. The positive benefits of zinc were not observed for the antitoxin immune responses; these were comparable between the supplemented and nonsupplemented children. There were no differences either between the responses (vibriocidal or antitoxic) in younger 6- to 9-month-old infants who most likely were not zinc deficient. The immunogenicity studies described excluded children who were severely malnourished ( $<3$ weight for age $\mathrm{Z}$ scores); however, many of the children were mildly or moderately malnourished ( $<1-3$ weight for age $\mathrm{Z}$ scores).

Recent studies on vaccine responses to Dukoral in Bangladeshi children have also revealed that breastfeeding may interfere with vaccine take. Children who were withheld from breastfeeding for $3 \mathrm{~h}$ before oral administration of the vaccine mounted more frequent vibriocidal immune responses than children who were only deprived from breastfeeding during $1 \mathrm{~h}$ before immunization [Ahmed et al., submitted]. However, it should be clearly recognized that breastfeeding per se has been strongly associated with substantial reduction in severe cholera. Thus, in studies during the 1980s, it was shown that breastfed children had a $70 \%$ reduced risk of developing severe cholera and that although the risk declined with age, it was clearly evident in children up to 30 months of age [62].

The killed WC cholera vaccine produced and used in Vietnam is very similar to Dukoral except that it includes cells from $V$. cholerae serotype O139, but does not contain the $B$ subunit [49]. Analysis of a trial of this vaccine found similar efficacy rates in children and adults, though the period of cholera risk was limited to 10 months, since the outbreak of cholera did not persist. The whole-cell vaccine appeared to be as immunogenic in children as in adults [63]. 


\section{Oral ETEC Vaccines}

The only ETEC vaccine that has been extensively tested in different age groups, including young children and infants, in developing countries consists of a combination of $\mathrm{rCTB}$ and formalin-inactivated ETEC bacteria expressing the most prevalent ETEC fimbriated colonization factors as well as some of the most prevalent $\mathrm{O}$ antigens [64]. Phase I and II trials of this oral rCTB-CF ETEC vaccine in Swedish, Bangladeshi and Egyptian adult volunteers have shown that the vaccine is well tolerated and gives rise to comparable mucosal immune responses in intestine (gut lavage fluid) or intestine-derived immune responses against the different vaccine antigens in a majority of the vaccinees from different settings. In analogous safety and immunogenicity clinical trials of the rCTB-CF ETEC vaccine in older children 2-9 years of age in developing countries, the vaccine was found to be equally immunogenic as in adults and also well tolerated, except in the youngest infants and toddlers; the latter groups mounted slightly lower immune responses than the older children [64-67].

The rCTB-CF ETEC vaccine has been tested for protective efficacy in placebo-controlled phase III vaccine trials in American travellers going to Mexico and Guatemala. In the first study encompassing nearly 700 volunteers, the vaccine was shown to provide significant protection (protective efficacy $77 \%$; $p=0.039$ ) against moderate to severe ETEC diarrheal illness, defined as symptoms that interfered with the travelers' daily activities or more than 5 loose stools in a day, but no significant protection against less severe ETEC diarrhea [68]. A subsequent similarly sized trial in the same setting also revealed that the vaccine protected against more severe symptoms in those vaccinees in which vaccine take could be documented [A.L. Bourgeois, pers. commun.]. However, when testing the efficacy of the rCTB-CF ETEC vaccine in a double-blind, placebo-controlled trial in 6- to 18 -month-old children in rural Egypt, there was no protection detected [S.J. Savarino, pers. commun.].

To evaluate if the lower responsiveness to the vaccine that was observed in infants in Egypt and Bangladesh may be explained by poor nutritional status, micronutrient deficiencies or other factors, studies are planned in Bangladesh to evaluate a possible influence of these different factors on the immune responses. These studies will focus especially on the mucosal immune responses when model oral vaccines (Dukoral and Vivotif) are administered with zinc supplements. Zinc effects the innate and adaptive (both humoral and cellular) immune re- sponses in children with ETEC diarrhea. Children with ETEC diarrhea, 6-24 months of age, who were given 20 mg of zinc for 10 days, showed a significantly higher complement C3 level in sera, higher phagocytic activity of cells as well as $\mathrm{T}$ cell responses compared with agematched children who were not supplemented. These results suggest that zinc supplementation may also be useful in improving ETEC-specific vaccine take rates and in decreasing the morbidity due to the disease [Larson and Qadri, pers. commun.].

\section{Oral Shigella Vaccines}

Only 1 live attenuated oral vaccine for shigellosis has been evaluated in a developing country. SC602 is a strain of Shigella flexneri 2a that is less virulent because of deletions of virulence plasmid gene icsA (mediating intraand intercellular spread) and chromosomal locus iuc (encoding aerobactin) [69]. In volunteers in the US, it was found to be safe, immunogenic, protective and excreted when used at a dose of $10^{4}$; however, it caused dysentery when used at higher doses $\left(10^{6}\right.$ or $\left.10^{8}\right)$ [69]. The vaccine was tested in Bangladeshi children at doses of 4, 5 and 6 logs. At these doses, there were no demonstrable side effects, and there were neither detectable serological responses nor excretion [70]. Due to the side effects seen with higher doses in the US, higher doses were not given to Bangladeshi children.

There is no clear understanding of the different immunogenicity responses between US volunteers and Bangladeshi children; however, the high requirement for iron related to the aerobactin deletion suggests a possible explanation. When grown in vitro, the strain requires additional iron in the media, but it will not grow when iron concentration is low. It seems possible that the concentrations of iron in the intestinal tract of the US volunteers and the Bangladeshi children may be quite different since Bangladeshi children have a diet that is very low in iron, while adults in the US regularly consume meat containing heme iron. It would seem likely that live oral bacterial vaccines which require colonization and multiplication, especially those given at relatively low doses, but also have a high iron requirement to multiply, may require buffers which provide this micronutrient. This may be especially true when the vaccine is intended for subjects whose diet is iron deficient. Studies analyzing if the provision of some supplemental iron might improve the immunogenicity of the vaccine strain have not been carried out. The importance of iron for bacterial infections 
is well established, and infection of a vaccine strain is not likely to be different in this respect from other types of bacterial infections. Because of the specific genetic mutation in this vaccine strain, it is likely even more dependent on the availability of iron. It cannot be ruled out that other nutritional deficiencies of the host as well as breastfeeding at the time of vaccination could have played a role for the poor vaccine take, but this will have to be tested in future controlled studies of live oral Shigella vaccine candidates.

Another possibility may be that the children in this setting had already developed some immunity to Shigella flexneri 2a-specific antigens by natural infection that may have protected against the low doses of the vaccine strain used. Though protection might be overcome by increasing the vaccine dose, such a strategy could also be risky for children without pre-existing immunity if the vaccine was reactogenic.

An injectable Shigella vaccine which utilizes an $\mathrm{O}$ polysaccharide conjugated to a protein was immunogenic and efficacious when tested in young adults in Israel [71]; however, this vaccine is not considered in this review as it is not given orally, and there are no data concerning its use in children in developing countries.

\section{Factors Influencing Immune Responses to Oral Vaccines in Developing Countries}

As highlighted above, many studies indicate that oral vaccines for polio, rotavirus, cholera, ETEC and Shigella may not be as immunogenic or as protective when given to subjects in developing countries. The evidence suggests that several factors may be responsible for this reduced immunogenicity. They include the following: (1) undernutrition, (2) micronutrient deficiencies including zinc, iron or vitamin A, (3) interference from breast milk antibody or maternal transplacental antibody, (4) interference from helminth infections, small bowel overgrowth with associated intestinal mucosal pathology, and (5) interference from intestinal, enterovirus infections. So far, there has been little speculation on the potential role for maternal malnutrition effecting the infant, but it is possible that maternal macro- or micronutrient malnutrition could reduce the ability of the infant's immune system to respond well to the vaccine antigens $[72,73]$.

In an attempt to sort through these factors which may result in reduced immunogenicity, age seems to be a critical variable. Factors that are important to the neonate or very young infant, e.g., breast milk or transplacental maternal antibody, may not be relevant to a child of 2 years. Vaccine responses for the older child are more likely to be reduced by undernutrition which is usually more pronounced in children above 1 year of age, often related to repeated diarrheal episodes during early childhood, small bowel overgrowth, mucosal pathology, helminth infections or micronutrient deficiency.

Although many studies have observed reduced immunogenicity among children in developing countries, unfortunately, there is no clear pattern indicating how to improve these responses. For example, to withhold breastfeeding for several hours during immunizations may effectively improve some vaccine responses, but this recommendation may not be accomplished easily in largescale programs. Deworming may also somewhat improve responses, which would seem to be a practical strategy, especially if it can be incorporated into immunization programs for children who are somewhat older. Zinc supplements are now recommended for children during episodes of diarrhea [74], and this may also benefit vaccine responsiveness, though it will need to be demonstrated in public health programs. Although routine supplements with vitamin A are now an important component of routine public health interventions, evidence from most vaccine studies suggests that additional vitamin A supplements are not likely to further enhance vaccine responses.

Live, attenuated bacterial vaccines may benefit by administering them with buffers designed to facilitate their temporary intestinal colonization. For example, a genetically engineered bacterial vaccine may have specific requirements for nutrients or minerals (e.g., iron), and these requirements might be included in the buffer in which the vaccine is given.

Importantly, additional information is needed to determine which conditions exist during fetal growth that may enhance or suppress the immune system of the infant and affect the response to vaccines given at an early age. It may be that certain micronutrients, e.g., zinc, if given to pregnant mothers, can improve vaccine immunogenicity for the infant.

In summary, one cannot assume that vaccines for children in developed countries will perform equally well when given to children in developing countries. They must be evaluated and optimized for these children to obtain their maximum benefit. 


\section{References}

1 Moore SE, Goldblatt D, Bates CJ, Prentice AM: Impact of nutritional status on antibody responses to different vaccines in undernourished Gambian children. Acta Paediatr 2003;92:170-176.

$\checkmark 2$ Faden H: Results of a clinical study of polio vaccine: the Buffalo experience. Pediatr Infect Dis J 1991;10:973-975.

- 3 Agarwal A, Sharma D, Kumari S, Khare S: Antibody response to three doses of standard and double dose of trivalent oral polio vaccine. Indian Pediatr 1991;28:1141-1145.

$\checkmark 4$ Sabin AB: Vaccination against poliomyelitis in economically underdeveloped countries. Bull World Health Organ 1980;58:141-157.

5 John TJ, Jayabal P: Oral polio vaccination of children in the tropics. 1 . The poor seroconversion rates and the absence of viral interference. Am J Epidemiol 1972;96:263-269.

-6 Sutter RW, Suleiman AJ, Malankar P, et al: Trial of a supplemental dose of four poliovirus vaccines. N Engl J Med 2000;343:767773.

7 Triki H, Abdallah MV, Ben AR, et al: Influence of host related factors on the antibody response to trivalent oral polio vaccine in Tunisian infants. Vaccine 1997;15:11231129.

-8 Swartz TA, Skalska P, Gerichter CG, Cockburn WC: Routine administration of oral polio vaccine in a subtropical area. Factors possibly influencing seroconversion rates. J Hyg (Lond) 1972;70:719-726.

$\checkmark 9$ Lepow ML, Warren RJ, Gray N, et al: Effect of Sabin type 1 poliomyelitis vaccine administered by mouth to newborn infants. $\mathrm{N} \mathrm{Engl}$ J Med 1961;264:1071-1078.

- 10 Plotkin SA, Katz M, Brown RE, Pagano JS: Oral poliovirus vaccination in newborn African infants. The inhibitory effect of breast feeding. Am J Dis Child 1966;111:27-30.

-11 Katz M, Plotkin SA: Oral polio immunization of the newborn infant; a possible method for overcoming interference by ingested antibodies. J Pediatr 1968;73:267-270.

-12 John TJ, Devarajan LV, Luther L, Vijayarathnam P: Effect of breast-feeding on seroresponse of infants to oral poliovirus vaccination. Pediatrics 1976;57:47-53.

13 Myaux JA, Unicomb L, Besser RE, et al: Effect of diarrhea on the humoral response to oral polio vaccination. Pediatr Infect Dis J 1996;15:204-209.

14 Bhaskaram P, Nair KM, Hemalatha P, et al: Systemic and mucosal immune response to polio vaccination with additional dose in newborn period. J Trop Pediatr 1997;43:232234.

15 Chopra K, Kundu S, Chowdhury DS: Antibody response of infants in tropics to five doses of oral polio vaccine. J Trop Pediatr 1989;35:19-23.
16 Bahl R, Bhandari N, Wahed MA, et al: Vitamin A supplementation of women postpartum and of their infants at immunization alters breast milk retinol and infant vitamin A status. J Nutr 2002;132:3243-3248.

17 Vesikari T, Isolauri E, d'Hondt E, et al: Protection of infants against rotavirus diarrhoea by RIT 4237 attenuated bovine rotavirus strain vaccine. Lancet 1984;i:977-981.

18 Hanlon P, Hanlon L, Marsh V, et al: Trial of an attenuated bovine rotavirus vaccine (RIT 4237) in Gambian infants. Lancet 1987;i: 1342-1345.

19 Georges-Courbot MC, Monges J, Siopathis MR, et al: Evaluation of the efficacy of a lowpassage bovine rotavirus (strain WC3) vaccine in children in Central Africa. Res Virol 1991;142:405-411.

20 De Mol P, Zissis G, Butzler JP, et al: Failure of live, attenuated oral rotavirus vaccine. Lancet 1986;ii:108.

21 Joensuu J, Koskenniemi E, Pang XL, Vesikari $\mathrm{T}$ : Randomised placebo-controlled trial of rhesus-human reassortant rotavirus vaccine for prevention of severe rotavirus gastroenteritis. Lancet 1997;350:1205-1209.

22 Rennels MB, Glass RI, Dennehy PH, et al: Safety and efficacy of high-dose rhesus-human reassortant rotavirus vaccines - report of the National Multicenter Trial. United States Rotavirus Vaccine Efficacy Group. Pediatrics 1996;97:7-13.

23 Lanata CF, Midthun K, Black RE, et al: Safety, immunogenicity, and protective efficacy of one and three doses of the tetravalent rhesus rotavirus vaccine in infants in Lima, Peru. J Infect Dis 1996;174:268-275.

24 Linhares AC, Gabbay YB, Mascarenhas JD, et al: Immunogenicity, safety and efficacy of tetravalent rhesus-human, reassortant rotavirus vaccine in Belem, Brazil. Bull World Health Organ 1996;74:491-500.

25 Perez-Schael I, Guntinas MJ, Perez M, et al: Efficacy of the rhesus rotavirus-based quadrivalent vaccine in infants and young children in Venezuela. N Engl J Med 1997;337: 1181-1187.

26 Linhares AC, Lanata CF, Hausdorff WP, et al: Reappraisal of the Peruvian and Brazilian lower titer tetravalent rhesus-human reassortant rotavirus vaccine efficacy trials: analysis by severity of diarrhea. Pediatr Infect Dis J 1999;18:1001-1006.

27 Ruiz-Palacios GM, Perez-Schael I, Velazquez FR, et al: Safety and efficacy of an attenuated vaccine against severe rotavirus gastroenteritis. N Engl J Med 2006;354:11-22.

28 Vesikari T, Matson DO, Dennehy P, et al: Safety and efficacy of a pentavalent humanbovine (WC3) reassortant rotavirus vaccine. N Engl J Med 2006;354:23-33.

29 Pichichero ME: Effect of breast-feeding on oral rhesus rotavirus vaccine seroconversion: a metaanalysis. J Infect Dis 1990;162: $753-755$.
30 Rennels MB, Wasserman SS, Glass RI, Keane VA: Comparison of immunogenicity and efficacy of rhesus rotavirus reassortant vaccines in breastfed and nonbreastfed children. US Rotavirus Vaccine Efficacy Group. Pediatrics 1995;96:1132-1136.

31 Linhares AC, Carmo KB, Oliveira KK, et al: Nutritional status in relation to the efficacy of the rhesus-human reassortant, tetravalent rotavirus vaccine (RRV-TV) in infants from Belem, Para state, Brazil. Rev Inst Med Trop Sao Paulo 2002;44:13-16

32 Perez-Schael I, Salinas B, Tomat M, et al: Efficacy of the human rotavirus vaccine RIX4414 in malnourished children. J Infect Dis 2007; 196:537-540.

-33 Lin FY, Ho VA, Khiem HB, et al: The efficacy of a Salmonella typhi $\mathrm{Vi}$ conjugate vaccine in two-to-five-year-old children. N Engl J Med 2001;344:1263-1269.

-34 Murphy JR, Grez L, Schlesinger L, et al: Immunogenicity of Salmonella typhi Ty21a vaccine for young children. Infect Immun 1991; 59:4291-4293.

35 Cryz SJ Jr, Vanprapar N, Thisyakorn U, et al: Safety and immunogenicity of Salmonella typhi Ty21a vaccine in young Thai children. Infect Immun 1993;61:1149-1151.

36 Cooper PJ, Chico M, Sandoval C, et al: Human infection with Ascaris lumbricoides is associated with suppression of the interleukin-2 response to recombinant cholera toxin B subunit following vaccination with the live oral cholera vaccine CVD 103-HgR. Infect Immun 2001;69:1574-1580.

37 Lagos R, Avendano A, Horwitz I, et al: Tolerance and immunogenicity of an oral dose of CVD 103-HgR, a live attenuated Vibrio cholerae 01 strain: a double-blind study of Chilean adults. Rev Med Chil 1993;121:857-863.

-38 Simanjuntak CH, O’Hanley P, Punjabi NH, et al: Safety, immunogenicity, and transmissibility of single-dose live oral cholera vaccine strain CVD $103-\mathrm{HgR}$ in 24 - to 59 month-old Indonesian children. J Infect Dis 1993;168:1169-1176.

39 Suharyono, Simanjuntak C, Witham N, et al: Safety and immunogenicity of single-dose live oral cholera vaccine CVD 103-HgR in 5-9-year-old Indonesian children. Lancet 1992;340:689-694

40 Richie EE, Punjabi NH, Sidharta YY, et al: Efficacy trial of single-dose live oral cholera vaccine CVD 103-HgR in North Jakarta, Indonesia, a cholera-endemic area. Vaccine 2000;18:2399-2410.

-41 Lagos R, Fasano A, Wasserman SS, et al: Effect of small bowel bacterial overgrowth on the immunogenicity of single-dose live oral cholera vaccine CVD 103-HgR. J Infect Dis 1999;180:1709-1712. 
-42 Sack DA, Sack RB, Shimko J, et al: Evaluation of Peru-15, a new live oral vaccine for cholera, in volunteers. J Infect Dis 1997;176:201205.

43 Cohen MB, Giannella RA, Bean J, et al: Randomized, controlled human challenge study of the safety, immunogenicity, and protective efficacy of a single dose of Peru-15, a live attenuated oral cholera vaccine. Infect Immun 2002;70:1965-1970.

44 Kenner JR, Coster TS, Taylor DN, et al: Peru15 , an improved live attenuated oral vaccine candidate for Vibrio cholerae O1. J Infect Dis 1995;172:1126-1129.

45 Qadri F, Chowdhury MI, Faruque SM, et al: Peru-15, a live attenuated oral cholera vaccine, is safe and immunogenic in Bangladeshi toddlers and infants. Vaccine 2007;25: 231-238.

-46 Sack DA, Shimko J, Sack RB, et al: Comparison of alternative buffers for use with a new live oral cholera vaccine, Peru-15, in outpatient volunteers. Infect Immun 1997;65: 2107-2111.

47 Clemens JD, Sack DA, Harris JR, et al: Field trial of oral cholera vaccines in Bangladesh. Lancet 1986;ii:124-127.

48 Holmgren J, Clemens J, Sack DA, Svennerholm AM: New cholera vaccines. Vaccine 1989;7:94-96.

-49 Trach DD, Clemens JD, Ke NT, et al: Field trial of a locally produced, killed, oral cholera vaccine in Vietnam. Lancet 1997;349: 231-235.

50 Clemens JD, Sack DA, Harris JR, et al: Field trial of oral cholera vaccines in Bangladesh: results from three-year follow-up. Lancet 1990;335:270-273.

51 Concha A, Giraldo A, Castaneda E, et al: Safety and immunogenicity of oral killed whole cell recombinant B subunit cholera vaccine in Barranquilla, Colombia. Bull Pan Am Health Organ 1995;29:312-321.

-52 Sanchez JL, Vasquez B, Begue RE, et al: Protective efficacy of oral whole-cell/recombinant-B-subunit cholera vaccine in Peruvian military recruits. Lancet 1994;344:12731276.
53 Taylor DN, Cardenas V, Sanchez JL, et al: Two-year study of the protective efficacy of the oral whole cell plus recombinant B subunit cholera vaccine in Peru. J Infect Dis 2000;181:1667-1673.

54 Lucas ME, Deen JL, von Seidlein L, et al: Effectiveness of mass oral cholera vaccination in Beira, Mozambique. N Engl J Med 2005; 352:757-767.

55 Taylor DN, Cardenas V, Perez J, et al: Safety, immunogenicity, and lot stability of the whole cell/recombinant B subunit (WC/ rCTB) cholera vaccine in Peruvian adults and children. Am J Trop Med Hyg 1999;61: 869-873.

56 Clemens JD, Stanton BF, Chakraborty J, et al: B subunit whole-cell and whole-cell only oral vaccines against cholera: studies on reactogenicity and immunogenicity. J Infect Dis 1987;155:79-85.

57 Sack DA, Clemens JD, Huda S, et al: Antibody responses after immunization with killed oral cholera vaccines during the 1985 vaccine field trial in Bangladesh. J Infect Dis 1991;164:407-411.

58 Hallander HO, Paniagua M, Espinoza F, et al: Calibrated serological techniques demonstrate significant different serum response rates to an oral killed cholera vaccine between Swedish and Nicaraguan children. Vaccine 2002;21:138-145.

59 Glass RI, Svennerholm AM, Stoll BJ, et al: Effects of undernutrition on infection with Vibrio cholerae $\mathrm{O} 1$ and on response to oral cholera vaccine. Pediatr Infect Dis J 1989;8: 105-109.

60 Albert MJ, Qadri F, Wahed MA, et al: Supplementation with zinc, but not vitamin A, improves seroconversion to vibriocidal antibody in children given an oral cholera vaccine. J Infect Dis 2003;187:909-913.

61 Qadri F, Ahmed T, Wahed MA, et al: Suppressive effect of zinc on antibody response to cholera toxin in children given the killed, B subunit-whole cell, oral cholera vaccine. Vaccine 2004;22:416-421.

62 Glass RI, Stoll BJ: The protective effect of human milk against diarrhea. A review of studies from Bangladesh. Acta Paediatr Scand Suppl 1989;351:131-136.

63 Trach DD, Cam PD, Ke NT, et al: Investigations into the safety and immunogenicity of a killed oral cholera vaccine developed in Viet Nam. Bull World Health Organ 2002; $80: 2-8$.
64 Svennerholm AM, Steele D: Microbial-gut interactions in health and disease. Progress in enteric vaccine development. Best Pract Res Clin Gastroenterol 2004;18:421-445.

65 Savarino SJ, Hall ER, Bassily S, et al: Introductory evaluation of an oral, killed whole cell enterotoxigenic Escherichia coli plus cholera toxin B subunit vaccine in Egyptian infants. Pediatr Infect Dis J 2002;21:322330.

66 Qadri F, Ahmed T, Ahmed F, et al: Reduced doses of oral killed enterotoxigenic Escherichia coli plus cholera toxin B subunit vaccine is safe and immunogenic in Bangladeshi infants 6-17 months of age: dosing studies in different age groups. Vaccine 2006;24:17261733.

67 Walker RI, Steele D, Aguado T: Analysis of strategies to successfully vaccinate infants in developing countries against enterotoxigenic E. coli (ETEC) disease. Vaccine 2007;25: 2545-2566.

68 Sack DA, Shimko J, Torres O, et al: Randomised, double-blind, safety and efficacy of a killed oral vaccine for enterotoxigenic $E$. coli diarrhoea of travellers to Guatemala and Mexico. Vaccine 2007;25:4392-4400.

69 Coster TS, Hoge CW, VanDeVerg LL, et al: Vaccination against shigellosis with attenuated Shigella flexneri 2a strain SC602. Infect Immun 1999;67:3437-3443

70 Anonymous: Future needs and directions for Shigella vaccines. Wkly Epidemiol Rec 2006; 81:51-58.

71 Cohen D, Ashkenazi S, Green MS, et al: Double-blind vaccine-controlled randomised efficacy trial of an investigational Shigella sonnei conjugate vaccine in young adults. Lancet 1997;349:155-159.

72 Wellinghausen N: Immunobiology of gestational zinc deficiency. Br J Nutr 2001;85 (suppl 2):S81-S86.

73 Golden MH, Jackson AA, Golden BE: Effect of zinc on thymus of recently malnourished children. Lancet 1977;ii:1057-1059.

74 Walker CF, Black RE: Zinc and the risk for infectious disease. Annu Rev Nutr 2004;24: 255-275. 